\title{
Protective effects of celastrol against $\gamma$ irradiation-induced oxidative stress in human umbilical vein endothelial cells
}

\author{
XIANG-BEI HAN ${ }^{1,2}$, YAN TAN ${ }^{2}$, YAN-QIU FANG ${ }^{2}$ and FENG LI ${ }^{3}$ \\ ${ }^{1}$ Department of Pathophysiology, Norman Bethune College of Medicine, Jilin University; \\ ${ }^{2}$ Tumor Biotherapy Center, Jilin Province People's Hospital; ${ }^{3}$ Department of Nursing, School of Nursing, \\ Jilin University, Changchun, Jilin 130021, P.R. China
}

Received December 14, 2017; Accepted May 31, 2018

DOI: $10.3892 /$ etm.2018.6270

\begin{abstract}
High-dose ionizing radiation can cause harmful effects on the cardiovascular system. Notably, endothelial cells are critical targets in radiation-induced damage. $\gamma$ radiation exerts its biological effects through the radiolysis of water, which further generates ROS and induces lipid peroxidation and DNA damage. The present study aimed to evaluate the potential protective effects of celastrol against $\gamma$ radiation-induced oxidative stress in human umbilical vein endothelial cells (HUVECs). HUVECs were exposed to $\gamma$ radiation at different doses with or without celastrol treatment. Cell viability and cytotoxicity, migratory ability, ROS production, lipid peroxidation, oxidative DNA damage and antioxidative enzyme levels were evaluated in HUVECs at $24 \mathrm{~h}$ post-irradiation. It was observed that HUVECs exhibited decreased cell viability, increased cytotoxicity and a decreased migratory ability after exposure to $20-\mathrm{Gy} \gamma$ radiation. Celastrol treatment concentration-dependently reversed these effects. $\gamma$ irradiation was also demonstrated to increase the production of ROS, enhance lipid peroxidation and oxidative DNA damage and decrease the levels of SOD, catalase, GST and GPx in HUVECs. These detrimental effects were blocked by treatment with celastrol for $24 \mathrm{~h}$. These data suggested that celastrol not only attenuated $\gamma$ radiation-induced cytotoxicity, but also effectively blocked oxidative stress in HUVECs. As an antioxidant agent, celastrol may have potential protective effects in HUVECs against $\gamma$ irradiation-induced injury.
\end{abstract}

\section{Introduction}

Cardiovascular disease is considered to be one of the most common non-cancer effects associated with high or medium doses of ionizing radiation. Endothelial cells, which are

Correspondence to: Professor Feng Li, Department of Nursing, School of Nursing, Jilin University, 965 Xinjiang Street, Changchun, Jilin 130021, P.R. China

E-mail: fenglijlu@outlook.com

Key words: $\gamma$ irradiation, celastrol, reactive oxygen species, antioxidant enzymes, human umbilical vein endothelial cells critical targets in radiation-induced cardiovascular damage, appear to serve a key role in the development of vascular pathologies. Increasing evidence has indicated that radiation exposure may induce premature senescence $(1,2)$, endothelial barrier damage or permeability changes $(1,3)$, cytoskeleton disruption (4) and angiogenic defects (5) in diverse endothelial models. Searching for an effective pharmacological therapy for radiation-induced cardiovascular damage has become an urgent task (6).

Although certain medicines, including pravastatin, have been proven to have the ability to reduce radiation-induced damage (7), considerable attention has been devoted to the development of radioprotectors from natural products in recent years, due to their cost-effectiveness and safety. Celastrol is a pentacyclic triterpenoid originally extracted from the root of the traditional Chinese medicinal plant Tripterygium wilfordii (also called Thunder god vine) $(8,9)$. Clinical trials have demonstrated the efficacy and safety of Tripterygium wilfordii. Tripterygium wilfordii has been applied in the treatment of rheumatoid arthritis $(10,11)$ and Crohn's disease $(12,13)$. Celastrol has also been proven to be effective in the treatment of asthma, chronic inflammation and neurodegenerative disease (14-17). The biological activities of celastrol include antioxidant (15), anti-inflammatory (18), anticancer (8), anti-diabetic (19), obesity-controlling (20) and insecticide (21) functions. To date, the function of celastrol in radiation protection has seldom been investigated.

$\gamma$ rays, a type of high-frequency ionizing radiation, can penetrate the body and cause the radiolysis of water. Human tissues contain $70-80 \%$ water. The major damage caused by $\gamma$ radiation results from free radicals, including ROS and nitric oxide (22). ROS react with cellular molecules, resulting in lipid peroxidation and DNA damage, and further causing cellular dysfunction and mortality. Given its properties of antioxidation, it was hypothesized that celastrol may exhibit protective effects against $\gamma$ irradiation-induced injury in human umbilical vein endothelial cells (HUVECs). The present study not only examined the protective effects of celastrol against $\gamma$ irradiation-induced cell death in HUVECs, but also explored the possible underlying mechanisms via the inhibition of oxidative stress. To the best of our knowledge, this is the first study to investigate the radioprotective properties of celastrol in endothelial cells. 


\section{Materials and methods}

Reagents. Celastrol, dimethyl sulfoxide (DMSO), Tris, glycine, sodium dodecyl sulfate (SDS), bovine serum albumin (BSA) and diphenyl-1-pyrenylphosphine (DPPP) were purchased from J\&K Scientific Ltd. (Beijing, China). 3-(4,5-dimethylthiazol-2-yl)-2,5-diphenyltetrazolium bromide (MTT), nuclease P1, alkaline phosphatase, $\beta$-actin antibody, dihydroethidium (DHE), a lipid peroxidation assay kit by malondialdehyde (MDA), CelLytic ${ }^{\mathrm{TM}}$ lysis reagent and Bradford reagents were obtained from Sigma-Aldrich Shanghai Trading Co. Ltd. (Shanghai, China). The Pierce Lactate Dehydrogenase (LDH) Cytotoxicity Assay kit was from Thermo Fisher Scientific,Inc.(Waltham,MA,USA). The 2',7'-dichlorofluorescin diacetate (DCFDA) cellular ROS detection assay kit, Alexa Fluor $^{\circledR}$ 647-conjugated anti-mouse antibody and Fluoroshield mounting medium with DAPI were purchased from Abcam Trade Ltd. (Shanghai, China). The genomic DNA purification kit and Griess reagent were purchased from Promega Biotech Co. Ltd. (Beijing China). EIA kits for 8-hydroxy-2-deoxy Guanosine (8-OH-dG), and antioxidant enzyme assays including superoxide dismutase (SOD), catalase, glutathione peroxidase (GPx) and glutathione S-transferase (GST) were purchased from Cayman Chemical Company (Ann Arbor, MI, USA). Antibodies against SOD-1, SOD-2, catalase, GPx and GST, and horseradish peroxidise (HRP)-conjugated goat anti-mouse and anti-rabbit antibodies were purchased from Santa Cruz Biotechnology Co. Ltd. (Shanghai, China). Anti- $\gamma \mathrm{H} 2 \mathrm{AX}$ antibody was from CST Biological Reagents Company Ltd. (Shanghai, China). The nitrocellulose membrane was obtained from Shanghai Xingya Purify Material Ltd. (Shanghai, China). Western blotting detection ECL Reagent was purchased from GE Healthcare China Co. Ltd. (Beijing, China).

Cell culture. HUVECs were purchased from AllCells Biotech Ltd. (Shanghai, China), and grown in endothelial cell growth medium (Jiangsu Promocell Biotechnology Co. Ltd., China) containing $2 \% \mathrm{FBS}$ at $37^{\circ} \mathrm{C}$ in a $5 \% \mathrm{CO}_{2}$ humidified atmosphere. Cells were passaged every three to four days. Cells at the 3rd to 8th passage were used for the present study.

Irradiation procedure and drug treatment. For all experiments, HUVECs were seeded in cell culture chamber slides, 96-well plates, $100 \mathrm{~mm}^{2}$ petri dishes or $75 \mathrm{~cm}^{2}$ cell culture flasks, and grown to confluence prior to being exposed to $\gamma$ radiation in a Gammacell 40 Exactor (Best Theratronics, Kanata, ON, Canada) using Cs-137 as a radioactive source. Different radiation doses were applied to generate the dose-response curve. The radiation dose rate was $2.8 \mathrm{~Gy} / \mathrm{min}$. Celastrol was dissolved in DMSO, kept at a stock solution of $100 \mathrm{mM}$, and further diluted in medium prior to administration. Following exposure to $\gamma$ irradiation, HUVECs were treated with celastrol at the designated concentrations for $24 \mathrm{~h}$, harvested and subjected to different assays.

Cell viability assay. The viability of HUVECs after $\gamma$ irradiation with or without celastrol treatment was tested using the colorimetric MTT method. The assay is based on the cleavage of the yellow tetrazolium salt MTT to purple formazan crystals by metabolically active cells. The formazan crystals are solubilized, and then spectrophotometrically quantified. Cells were seeded in 96-well plates, exposed to $\gamma$ irradiation at different doses, and then incubated in medium containing different concentrations of celastrol. After $24 \mathrm{~h}$ of treatment, MTT at a final concentration of $0.5 \mathrm{mg} / \mathrm{ml}$ was added to each well and incubated at $37^{\circ} \mathrm{C}$ for $4 \mathrm{~h}$. When the medium had been removed, HUVECs were washed twice with PBS. DMSO was added into the wells to solubilize the blue formazan dye and the absorbance was read at $570 \mathrm{~nm}$. Cells without $\gamma$ irradiation exposure and celastrol treatment were considered to be controls. The cell viability of the treatment groups is expressed as a percentage of the control.

Cell cytotoxicity assay. Cytotoxicity induced by $\gamma$ irradiation was determined by LDH release. As a cytosolic enzyme present in many different types of cells, LDH is released into the cell culture medium when the plasma membrane is damaged. Cells were seeded in 96-well plates and exposed to $20 \mathrm{~Gy} \gamma$ irradiation, followed by the treatment with 1.5 and $2 \mu \mathrm{M}$ celastrol for $24 \mathrm{~h}$. A volume of $50 \mu \mathrm{l}$ medium was loaded into a 96-well flat bottom plate in triplicate wells, and $50 \mu 1$ reaction mixture was added to each well. The plate was incubated at room temperature for $30 \mathrm{~min}$ in the dark and $50 \mu \mathrm{l}$ stop solution was added. The absorbance at $490 \mathrm{~nm}$ was measured using a microplate reader (Tecan Group, Ltd., Männedorf, Switzerland). The cytotoxicity in each group was expressed as a percentage of the control.

Cell migration assay. Cells were seeded in Culture-Insert 2 wells in $\mu$-Dish $35 \mathrm{~mm}$ (cat. no. 81176; ibidi $\mathrm{GmbH}$, Am Klopferspitz, Planegg, Germany) with a defined $500 \mu \mathrm{m}$ cell-free gap. Following exposure to 20-Gy $\gamma$ irradiation, cell medium was removed and cells were treated with 1.5 and $2 \mu \mathrm{M}$ celastrol dissolved in culture medium for $24 \mathrm{~h}$. The silicone inserts were carefully removed, and the dishes were monitored under an inverted microscope (Olympus Corp., Tokyo, Japan). The gap distances were measured at different time points. The data at $6 \mathrm{~h}$ after the removal of the inserts was used for comparison.

Measurement of ROS. The intracellular level of ROS was measured using the fluorescent probe $\mathrm{H}_{2}$ DCFDA and DHE. DHE, upon reaction with superoxide anions, forms a red fluorescent product, 2-hydroxyethidium. HUVECs were seeded in black-walled and clear-bottom 96-well microplates (cat. no. M33089) and Nunc ${ }^{\text {TN }}$ Lab-Tek ${ }^{\text {TM }}$ Chambered Coverglass (cat. no. 155383; both Thermo Fisher Scientific, Inc.). After exposure to 20-Gy $\gamma$ irradiation and treatment with 1.5 and $2 \mu \mathrm{M}$ celastrol, cells were washed with PBS and stained with $5 \mu \mathrm{M}$ DHE at $37^{\circ} \mathrm{C}$ for $30 \mathrm{~min}$ in the dark. Images of the coverglass were captured under a Leica fluorescent microscope (Leica Biosystems, Wetzlar, Germany). The fluorescence intensities in the 96-well plate were measured using a fluorescent microplate reader (Tecan Infinite F200 Pro; Tecan Group, Ltd.) at excitation and emission wavelengths of 520 and $610 \mathrm{~nm}$, respectively.

ROS production was also examined by $\mathrm{H}_{2}$ DCFDA staining. As a cell-permeant and non-fluorescent probe, $\mathrm{H}_{2}$ DCFDA is converted to the highly fluorescent 2 ', $7^{\prime}$-dichlorofluorescein (DCF) upon cleavage of the acetate groups by intracellular esterases and oxidation. Cells were treated as stated above and 


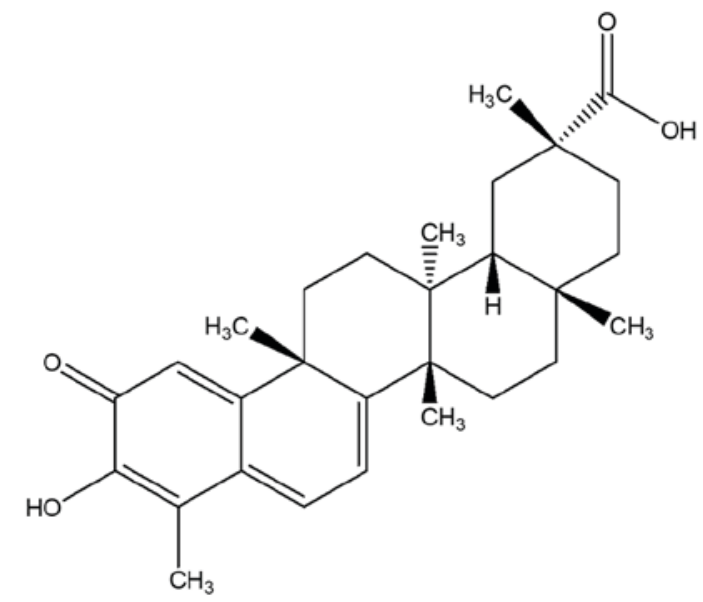

Figure 1. Chemical structure of celastrol, a pentacyclic triterpenoid.

stained with $25 \mu \mathrm{M} \mathrm{H}_{2}$ DCFDA at $37^{\circ} \mathrm{C}$ for $30 \mathrm{~min}$ in the dark. The fluorescence intensities were measured at excitation and emission wavelengths of 485 and $530 \mathrm{~nm}$, respectively.

Lipid peroxidation assays. Lipid peroxidation was examined using DPPP, a probe which turns to a fluorescent diphenyl-1-pyrenylphosphine oxide (DPPP-O) when reacting with hydroperoxides. HUVECs were seeded in 96-well plates, treated as stated above, and incubated with $50 \mu \mathrm{M}$ DPPP at $37^{\circ} \mathrm{C}$ for $60 \mathrm{~min}$ in the dark. The fluorescence intensities were measured and analyzed at an excitation wavelength of $351 \mathrm{~nm}$ and an emission wavelength of $380 \mathrm{~nm}$ using a fluorescent microplate reader.

Lipid peroxidation was also evaluated according to the levels of MDA. A total of $1 \times 10^{6}$ cells were homogenized on ice in $300 \mu \mathrm{l}$ MDA lysis buffer containing $3 \mu \mathrm{l}$ butylated hydroxytoluene (BHT; 100X) and centrifuged at $13,000 \mathrm{x}$ g for $10 \mathrm{~min}$ to remove insoluble material. A total of $200 \mu \mathrm{l}$ supernatant was removed into a microcentrifuge tube. A total of $600 \mu \mathrm{l}$ thiobarbituric acid (TBA) solution was added into each tube containing the standard and samples, and incubated at $95^{\circ} \mathrm{C}$ for $60 \mathrm{~min}$. Following cooling to room temperature, $200 \mu \mathrm{l}$ from each reaction mixture was pipetted to a 96-well plate for analysis. The absorbance at $490 \mathrm{~nm}$ was measured with a microplate reader. The concentration of TBA-MDA adduct was calculated based upon the standard curve.

DNA oxidative damage assay using an 8-OH-dG EIA kit. HUVECs were seeded in $100 \mathrm{~mm}^{2}$ Petri dishes and treated as stated above. Genomic DNA was isolated and purified with a commercial kit. HUVECs were harvested by trypsinization, and treated with RNase $\mathrm{A}$, proteinase $\mathrm{K}$ and lysis buffer at $55^{\circ} \mathrm{C}$ for $10 \mathrm{~min}$. The lysate was loaded into the separation column. After the column was centrifuged and washed, the eluted and purified DNA was collected.

An 8-OH-dG EIA kit was used to examine DNA oxidative damage in cells according to the manufacturer's instructions. Briefly, the aforementioned purified DNA was incubated with alkaline phosphatase and nuclease $\mathrm{P} 1$ at $37^{\circ} \mathrm{C}$ for $30 \mathrm{~min}$, and then boiled for $10 \mathrm{~min}$ before being placed on ice. A total of $50 \mu 1$ sample or standard, $50 \mu 1$ antibodies and $50 \mu 1$ Tracer were added into the testing plate, which was then incubated at $4^{\circ} \mathrm{C}$ for $18 \mathrm{~h}$. A total of $200 \mu \mathrm{l}$ Ellman's reagent was added into the plate following washing. The plate was shaken for 90-120 min in the dark for optimum development. The absorbance was measured using a microplate reader at a wavelength of $420 \mathrm{~nm}$. The reading was used to calculate the $8-\mathrm{OH}-\mathrm{dG}$ concentration based upon the standard curve.

DNA double-strand break assay by immunofluorescence staining. DNA double-strand breaks were studied using anti- $\gamma \mathrm{H} 2 \mathrm{AX}$ immunofluorescence staining. Briefly, HUVECs were grown on chamber slides and treated as described above. Cells were fixed in cold methanol for $10 \mathrm{~min}$, washed with PBS three times, blocked using $1 \%$ BSA for $1 \mathrm{~h}$ at room temperature and incubated with primary anti- $\gamma \mathrm{H} 2 \mathrm{AX}$ antibody (1:200 dilution) at $4^{\circ} \mathrm{C}$ overnight. Following washing, cells were incubated with secondary Alexa Fluor 647-conjugated anti-mouse antibody (1:200 dilution) for $1 \mathrm{~h}$ at room temperature. Following rinsing, the nuclei were fluorescently labelled with DAPI in mounting medium. The images were examined under a fluorescence microscope.

Antioxidant enzyme activity assay. HUVECs were treated, harvested using a rubber policeman, and centrifuged at $2,000 \mathrm{x} \mathrm{g}$ for $10 \mathrm{~min}$. The cell pellets were lysed in cold lysis buffer, and the protein supernatants were collected by centrifugation at $10,000 \mathrm{x} \mathrm{g}$ at $4^{\circ} \mathrm{C}$ for $15 \mathrm{~min}$. The protein concentration was evaluated using the Bradford method. Equal amounts of samples and standards were loaded into the 96-well plates provided with the commercial kits. The concentrations and activities of SOD, catalase, GST and GPx were measured according to the respective protocols from the manufacturer. The absorbance was read using a microplate reader, and the activities of SOD, catalase, GST and GPx were calculated using the equations obtained from their respective standard curves.

Immunoblot analysis. Cell lysates were separated on a $10 \%$ SDS-PAGE gel and transferred to a nitrocellulose membrane. The membranes were blocked using 5\% BSA and incubated with the respective primary antibodies ( $\beta$-actin, 1:1,000 dilution; SOD-1, SOD-2, GPx, GST and catalase, 1:500 dilution) overnight at $4^{\circ} \mathrm{C}$. Following washing, the membrane was incubated with the respective HRP-conjugated secondary antibodies (1:10,000 dilution) at room temperature for $1 \mathrm{~h}$, and visualized using the ECL method. Images were captured and quantified using the Bio-Rad Laboratories, Inc. Gel Doc system. Values were normalized to their respective loading control. The results were calculated and expressed as a fold change relative to control group.

Statistical analysis. Values which were normally distributed are expressed as the mean \pm SEM. One-way analysis of variance (ANOVA) was used to determine statistical significance between groups, followed by Tukey's post-hoc test. P 0.05 was considered to indicate a statistically significant difference.

\section{Results}

Effects of celastrol on cell viability and cytotoxicity in HUVECs following $\gamma$ irradiation. The chemical structure of 
celastrol is presented in Fig. 1. HUVECs were exposed to $\gamma$ irradiation at different doses. It was observed that 10-, 20- and 40-Gy $\gamma$ irradiation significantly decreased the cell viability at $24 \mathrm{~h}$ post-irradiation, indicative of a dose-dependent response (Fig. 2A). Treatment of HUVECs with $1,1.5$ and $2 \mu \mathrm{M}$ celastrol for $24 \mathrm{~h}$ following $20-\mathrm{Gy} \gamma$ radiation exposure significantly reversed the decreased cell viability (Fig. 2B), while treatment with celastrol at $0.5 \mu \mathrm{M}$ did not exert any effect.

The protective effect of celastrol against $\gamma$ irradiation-induced cytotoxicity was also supported by the LDH release assay (Fig. 2C). Compared with the control, 20-Gy $\gamma$ irradiation significantly induced LDH release into the medium, while treatment with 1.5 and $2 \mu \mathrm{M}$ celastrol significantly decreased LDH release to the level of the control group. These results indicated that celastrol could reverse $\gamma$ irradiation-induced cell death, indicating the radioprotective ability of celastrol.

Effects of celastrol on the decreased migratory ability of HUVECs following $\gamma$ irradiation. A gap closure assay was used to examine the effects of celastrol on the migratory potential of HUVECs following $\gamma$ irradiation. It was observed that 20-Gy $\gamma$ irradiation significantly inhibited the migratory ability of HUVECs, indicated by the increased gap distance when compared with the control (Fig. 2D and E). Cells following treatment with celastrol at the concentrations of 1.5 and $2 \mu \mathrm{M}$ exhibited a decreased gap distance, suggesting the restored migratory ability of HUVECs (Fig. 2D and E).

Effects of celastrol on the increased free radical production induced by $\gamma$ irradiation. DHE staining illustrated elevated red fluorescence intensity at $24 \mathrm{~h}$ post-exposure to 20-Gy $\gamma$ irradiation in HUVECs (Fig. 3A and B), indicating increased ROS production. This increase was significantly blocked by treatment with celastrol at 1.5 and $2 \mu \mathrm{M}$.

ROS production was also evaluated by $\mathrm{H}_{2}$ DCFDA. Similar results were observed, in terms of the enhanced ROS production following $\gamma$ radiation exposure which was reversed by celastrol treatment at 1.5 and $2 \mu \mathrm{M}$ for $24 \mathrm{~h}$ (Fig. 3C). These results suggested that the protective effects of celastrol against $\gamma$ irradiation-induced injury in HUVECs are mediated by the inhibition of oxidative stress.

Effects of celastrol on the increased lipid peroxidation induced by $\gamma$ irradiation. ROS may induce the oxidative degradation of lipids in cell membranes, resulting in cell damage. Lipid peroxidation was observed to increase in HUVECs at $24 \mathrm{~h}$ post-irradiation, indicated by a higher fluorescence intensity of DPPP compared with the control (Fig. 3D), while treatment with celastrol at 1.5 and $2 \mu \mathrm{M}$ for $24 \mathrm{~h}$ significantly blocked this increase. The ability of celastrol to protect the cells against $\gamma$ irradiation-induced lipid peroxidation was also evaluated by MDA assay (Fig. 3E). As the end-product of lipid peroxidation, MDA is known to be a major bioactive marker of lipid peroxidation. The TBS-MDA concentration was significantly higher in HUVECs following exposure to 20-Gy $\gamma$ irradiation in comparison with the control. This enhancement was blocked by treatment with celastrol at 1.5 and $2 \mu \mathrm{M}$ for $24 \mathrm{~h}$, suggesting the protective effects of celastrol in HUVECs against cell damage from lipid peroxidation.
Effects of celastrol on the increased oxidative DNA damage induced by $\gamma$ irradiation. $8-\mathrm{OH}-\mathrm{dG}$ is probably the most representative product of oxidative modifications of DNA, and thus is widely used as a non-invasive biomarker of oxidative damage to DNA. Increased 8-OH-dG concentrations were observed in HUVECs following exposure to 20-Gy $\gamma$ irradiation, suggesting elevated oxidative DNA damage (Fig. 4A). The enhancement was significantly blocked by $2 \mu \mathrm{M}$ celastrol treatment for $24 \mathrm{~h}$. Treatment with $1.5 \mu \mathrm{M}$ celastrol in HUVECs also decreased the 8-OH-dG concentration when compared with that in the $20 \mathrm{~Gy}$ group; however, the difference was not statistically significant (Fig. 4A).

Oxidative stress has also been proposed to be responsible for the production of DNA strand breaks. $\gamma \mathrm{H} 2 \mathrm{AX}$ is widely used as a marker of DNA double-strand breaks. Fig. 4B presents fluorescence images of $\gamma \mathrm{H} 2 \mathrm{AX}$ in HUVECs, and the number of $\gamma \mathrm{H} 2 \mathrm{AX}$ foci in the cell nuclei is displayed in Fig. 4C. It was demonstrated that compared with the control, the exposure to 20-Gy $\gamma$ irradiation significantly increased the frequency of DNA double-strand breaks, while celastrol treatment at 1.5 and $2 \mu \mathrm{M}$ for $24 \mathrm{~h}$ blocked this enhancement (Fig. 4B and C). The above results indicated the protective activity of celastrol against $\gamma$ irradiation-induced oxidative DNA damage.

Effects of celastrol on the activity and protein expression of antioxidant enzymes. The activities of antioxidant enzymes are presented in Table I. 20-Gy $\gamma$ irradiation significantly decreased the activities of SOD, catalase, GPx and GST compared with the control, while treatment with celastrol at $1.5 \mu \mathrm{M}$ for $24 \mathrm{~h}$ significantly elevated the activities of these antioxidant enzymes.

In order to validate the findings, we further investigated the protein expression of SOD-1, SOD-2, catalase, GPx and GST in HUVECs (Fig. 5). The expression levels of all antioxidant enzymes decreased in HUVECs following exposure to 20-Gy $\gamma$ irradiation as compared to the control group. Treatment with celastrol significantly increased the expression levels of these antioxidant enzymes when compared to the 20 Gy group. The expression of SOD-1, catalase and GPx was restored to the levels of the control groups, but celastrol treatment only partially enhanced the protein expression of SOD-2 and GST (Fig. 5B). These western blot data were in line with the results of the corresponding enzyme activity assays. Taken together, these data indicated that celastrol may enhance the activities of antioxidative systems in HUVECs following $\gamma$ irradiation.

\section{Discussion}

Humans are exposed to ionizing radiation from electronic devices, air travel, diagnostic and therapeutic procedures, and even nuclear accidents. The increased use of nuclear energy has made the search for safe and effective radioprotective agents a priority (23). Although previous studies have examined the protective effects of certain synthetic pharmaceutical agents, including atorvastatin and recilisib sodium, against radiation induced injury $(24,25)$, compounds from natural sources have still become the potential targets due to their pharmacological properties and decreased toxicity (26). Podophyllum hexandrum (Himalayan mayapple) was observed to exhibit radioprotective effects in lethally 



E

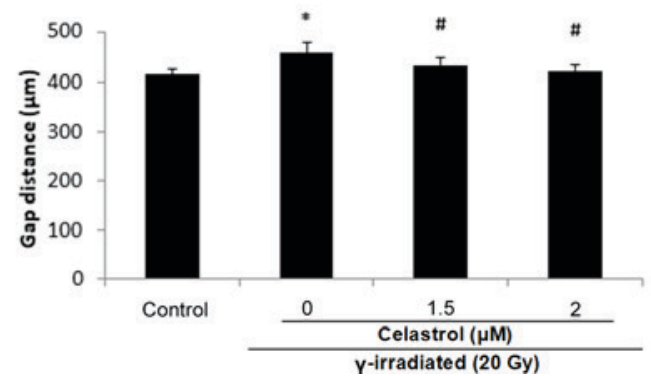

Figure 2. Cell viability, cytotoxicity and migratory ability of HUVECs following exposure to $\gamma$ irradiation of different doses and celastrol treatment at different concentrations. (A) HUVECs were exposed to $\gamma$ irradiation of 5 to $40 \mathrm{~Gy}$. Cell viability was evaluated by MTT after $24 \mathrm{~h}$. (B) HUVECs were exposed to 20-Gy $\gamma$ irradiation, followed by treatment with celastrol at various concentrations $(0,0.5,1,1.5$ and $2 \mu \mathrm{M})$. Cell viability was evaluated by MTT after $24 \mathrm{~h}$. (C) Cells were exposed to 20-Gy $\gamma$ irradiation, followed by treatment with celastrol at 1.5 and $2 \mu \mathrm{M}$. Cytotoxicity was determined by LDH release. HUVECs without celastrol treatment and $\gamma$ radiation served as controls. Cell viability and cytotoxicity are expressed as a percentage of the control. (D) Images of HUVECs in the cell migration assay at $6 \mathrm{~h}$ after the removal of the inserts. The dishes were monitored under an inverted microscope (magnification, $\mathrm{x} 40$ ). (E) Statistical results of the cell migration assay. The gap distances at $6 \mathrm{~h}$ after the removal of insert were used for comparison. All data are expressed as the mean \pm SEM ANOVA was used to determine statistical significance between groups followed by Tukey's post-hoc test. " $\mathrm{P}<0.05$ vs. control; " $\mathrm{P}<0.05$ vs. $20 \mathrm{~Gy}$ without celastrol treatment group. HUVECs, human umbilical vein endothelial cells; MTT, 3-(4,5-dimethylthiazol-2-yl)-2,5-diphenyltetrazolium bromide; LDH, Lactate Dehydrogenase; ANOVA, one-way analysis of variance.

irradiated mice $(27,28)$. Its extracts, containing several active components, have exhibited antioxidant activity as indicated by the inhibition of nitric oxide production and the promotion of DNA repair $(29,30)$. Acorus calamus (sweet flag or calamus, from the Acoraceae family) and its extracts were proven to scavenge free radicals and enhance the activities of antioxidant enzymes in mouse liver homogenates, and in mice exposed to $\gamma$ irradiation $(31,32)$. These studies suggested the radioprotective potential of natural products against radiation-induced damages. However, the existence of multiple bioactive components in these plant extracts impedes investigations into the underlying molecular and biochemical mechanisms.

Therefore, celastrol was used in the present study, its potential efficacy in the protection against $\gamma$ irradiation-induced cell injury was examined. To date, no study has been conducted on the structure-activity association of celastrol in radioprotection, to the best of our knowledge. The hydroxyl group at position C-3 and the carboxylic group at position C-20 of celastrol are believed to serve important roles in its activities (33). It was demonstrated in the present study that $\gamma$ irradiation significantly decreased cell viability and increased cytotoxicity in
HUVECs, while this effect was reversed by treatment with celastrol at 1 and $2 \mu \mathrm{M}$, indicative of a dose-dependent effect of celastrol against $\gamma$ irradiation-induced cell death. To date, very little evidence has been published on the ability of celastrol to prevent $\gamma$ radiation-induced injury, although its effects in controlling the growth of various cancer cells have been recognized (34). It has been proven that celastrol is able to inhibit the migration and invasion of ovarian cancer cells by blocking the NF- $\mathrm{kB}$ pathway (35). Nevertheless, the present study is the first to assess the protective activities of celastrol on $\gamma$ radiation-induced damage in HUVECs.

Endothelial cells serve an important role in maintaining endothelial integrity.Previous studies have suggested a causality between high-dose radiation exposure and the development of cardiovascular disease (36). Endothelial cells, as the most sensitive cell type in the vasculature, are undoubtedly critical targets in radiation-induced cardiovascular damage. In the present study, the migratory ability of HUVECs was observed to be inhibited by 20-Gy $\gamma$ irradiation. The present results were consistent with those from a study form Hwang et al (37), who demonstrated that far-infrared radiation inhibited the 
A

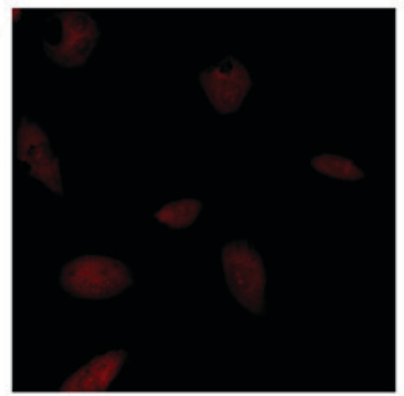

Control

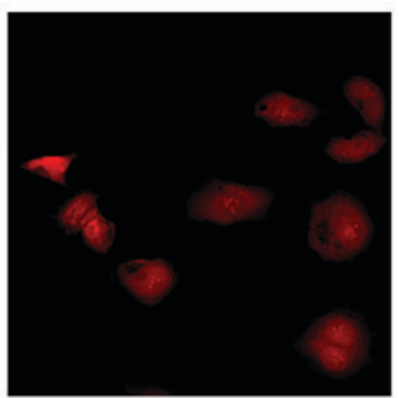

20 Gy

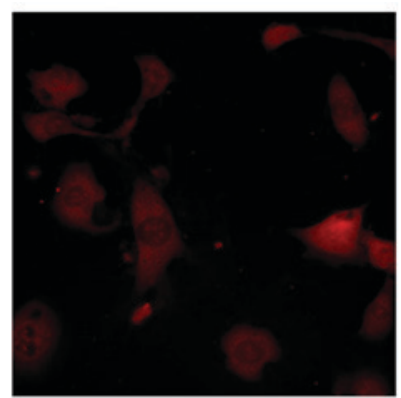

$1.5 \mu \mathrm{M}$ celastrol

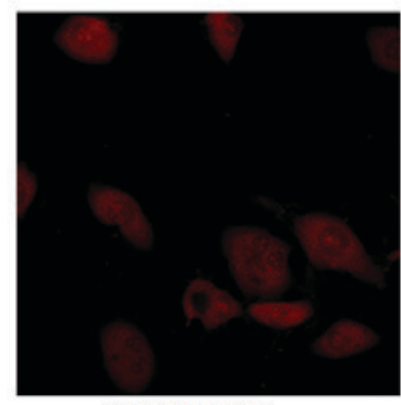

$2.0 \mu \mathrm{M}$ celastrol
B

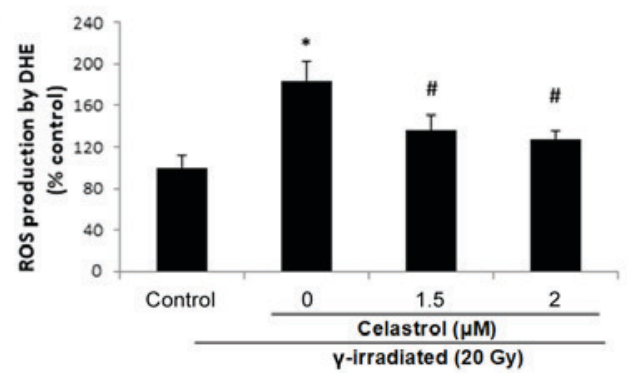

D

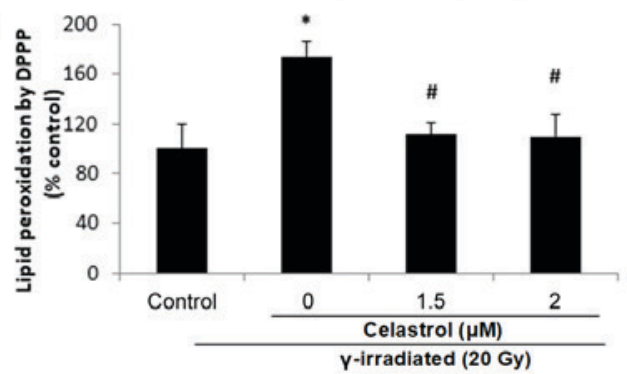

C

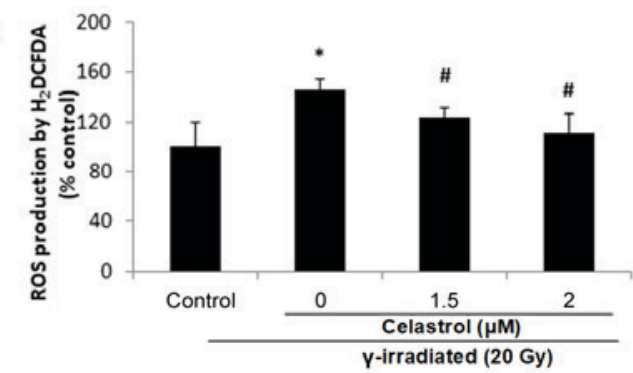

E

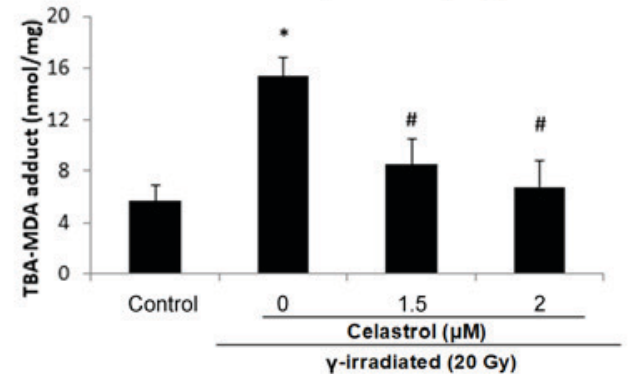

Figure 3. Effects of celastrol on ROS production and lipid peroxidation in HUVECs. Cells were exposed to 20 -Gy $\gamma$ irradiation, followed by treatment with 1.5 and $2 \mu \mathrm{M}$ celastrol. HUVECs without celastrol treatment and $\gamma$ radiation served as the control. (A) Images of DHE staining in HUVECs under a fluorescence microscope (magnification, $\mathrm{x} 400$ ). (B) Statistical results of ROS production analysed by DHE. HUVECs were stained with $5 \mu \mathrm{M}$ DHE at $37^{\circ} \mathrm{C}$ for 30 min in the dark. The fluorescence intensities of DHE were viewed and measured an excitation and emission wavelength of 520 and 610 nm, respectively. (C) ROS production in HUVECs assayed by $25 \mu \mathrm{M} \mathrm{H}_{2}$ DCFDA. The fluorescence intensities of $\mathrm{H}_{2}$ DCFDA were measured at an excitation and emission wavelength of 485 and $530 \mathrm{~nm}$, respectively. (D) Lipid peroxidation assayed by $50 \mu \mathrm{M}$ DPPP. The fluorescence intensities of DPPP fluorescence were measured at an excitation wavelength of $351 \mathrm{~nm}$ and an emission wavelength of $380 \mathrm{~nm}$. (E) Lipid peroxidation examined by MDA in HUVECs. The concentrations of the TBA-MDA adduct were determined by the absorbance at $490 \mathrm{~nm}$ based upon the standard curve. All data are expressed as the mean \pm SEM. ANOVA was used to determine statistical significance between groups followed by Tukey's post-hoc test. *P<0.05 vs. control; ${ }^{*} \mathrm{P}<0.05$ vs. 20 Gy without celastrol treatment group. HUVECs, human umbilical vein endothelial cells; DHE, dihydroethidium; H 2 DCFDA, 2',7'-dichlorofluorescin diacetate; MDA, malondialdehyde; DPPP, diphenyl-1-pyrenylphosphine; ANOVA, one-way analysis of variance.

proliferation, migration and angiogenesis of HUVECs. The attenuated migratory ability of HUVECs was restored by treatment with celastrol. The application of effective agents to prevent injury in endothelial cells may have substantial radioprotective effects against cardiovascular disease.

It is accepted today that oxidative stress is a potent pathogenic mechanism contributing to $\gamma$ radiation-induced damage $(18,38)$. Recent studies have proposed that $\gamma$ radiation significantly increases intracellular ROS formation, and intracellular MDA and LDH levels, and decreases the production of the intracellular antioxidants glutathione (GSH) and SOD in endothelial cells $(39,40)$. Our results indicated that 20-Gy $\gamma$ radiation significantly elevated the production of ROS and the induction of lipid peroxidation. It is believed that ionizing irradiation exerts its biological effects by initially generating ROS. ROS then react with unsaturated lipids, alter membrane permeability and induce lipid peroxidation (38). A study by $\mathrm{Hu}$ et al (41) demonstrated that acute $\gamma$ radiation dose-dependently increased intracellular ROS levels in
HUVECs at $24 \mathrm{~h}$ post-irradiation. ROS and MDA production was found to be enhanced after UVB exposure in HUVECs, suggesting the oxidative effects of ionizing radiation on membrane lipids. Single or fractionated irradiations with low-dose X-rays were demonstrated to induce ROS generation in HUVECs, even when these irradiations did not affect cell viability and DNA repair (42). ROS may also cause the progressive modification of cellular DNA. Such cumulative and deleterious effects induced by ROS can lead to cell function loss and cell death. Our study showed that $\gamma$ irradiation not only increases the concentration of $8-\mathrm{OH}-\mathrm{dG}$ in HUVECs, but also induces more DNA double strand breaks. This finding was consistent with other literature. Olteanu et al (43) demonstrated that UVB exposure induced DNA damage, as indicated by the increased expression of $\gamma-\mathrm{H}_{2} \mathrm{AX}$ in HUVECs, accompanied by increased apoptosis and cell death. Chronic low-dose ionizing radiation was also demonstrated to induce DNA damage and oxidative stress in HUVECs (2). All of this evidence not only supports our finding that 20-Gy 
Table I. Antioxidant enzyme activities in study groups.

\begin{tabular}{lcccc}
\hline Group & $\begin{array}{c}\text { Superoxide dismutase } \\
(\mathrm{U} / \mathrm{mg})\end{array}$ & $\begin{array}{c}\text { Catalase } \\
(\mathrm{nmol} / \mathrm{min} / \mathrm{mg})\end{array}$ & $\begin{array}{c}\text { Glutathione S-transferase } \\
(\mathrm{nmol} / \mathrm{min} / \mathrm{mg})\end{array}$ & $\begin{array}{c}\text { Glutathione peroxidase } \\
(\mathrm{nmol} / \mathrm{min} / \mathrm{mg})\end{array}$ \\
\hline Control & $3.23 \pm 0.85$ & $20.37 \pm 2.32$ & $73.82 \pm 5.98$ & $58.64 \pm 7.35$ \\
$20 \mathrm{~Gy}$ & $2.15 \pm 0.17^{\mathrm{a}}$ & $13.26 \pm 2.24^{\mathrm{a}}$ & $42.26 \pm 3.12^{\mathrm{a}}$ & $30.23 \pm 4.12^{\mathrm{a}}$ \\
Celastrol+20 Gy & $3.03 \pm 0.48^{\mathrm{b}}$ & $21.25 \pm 3.39^{\mathrm{b}}$ & $65.56 \pm 6.01^{\mathrm{a}, \mathrm{b}}$ & $46.26 \pm 3.54^{\mathrm{a}, \mathrm{b}}$ \\
\hline
\end{tabular}

All data was expressed as mean \pm standard error of the mean. ANOVA was used to determine statistical significance between groups followed by Tukey's post-hoc test. ${ }^{\mathrm{P}} \mathrm{P}<0.05$ vs. control; ${ }^{\mathrm{b}} \mathrm{P}<0.05$ vs. $20 \mathrm{~Gy}$ without celastrol treatment group. ANOVA, one-way analysis of variance.
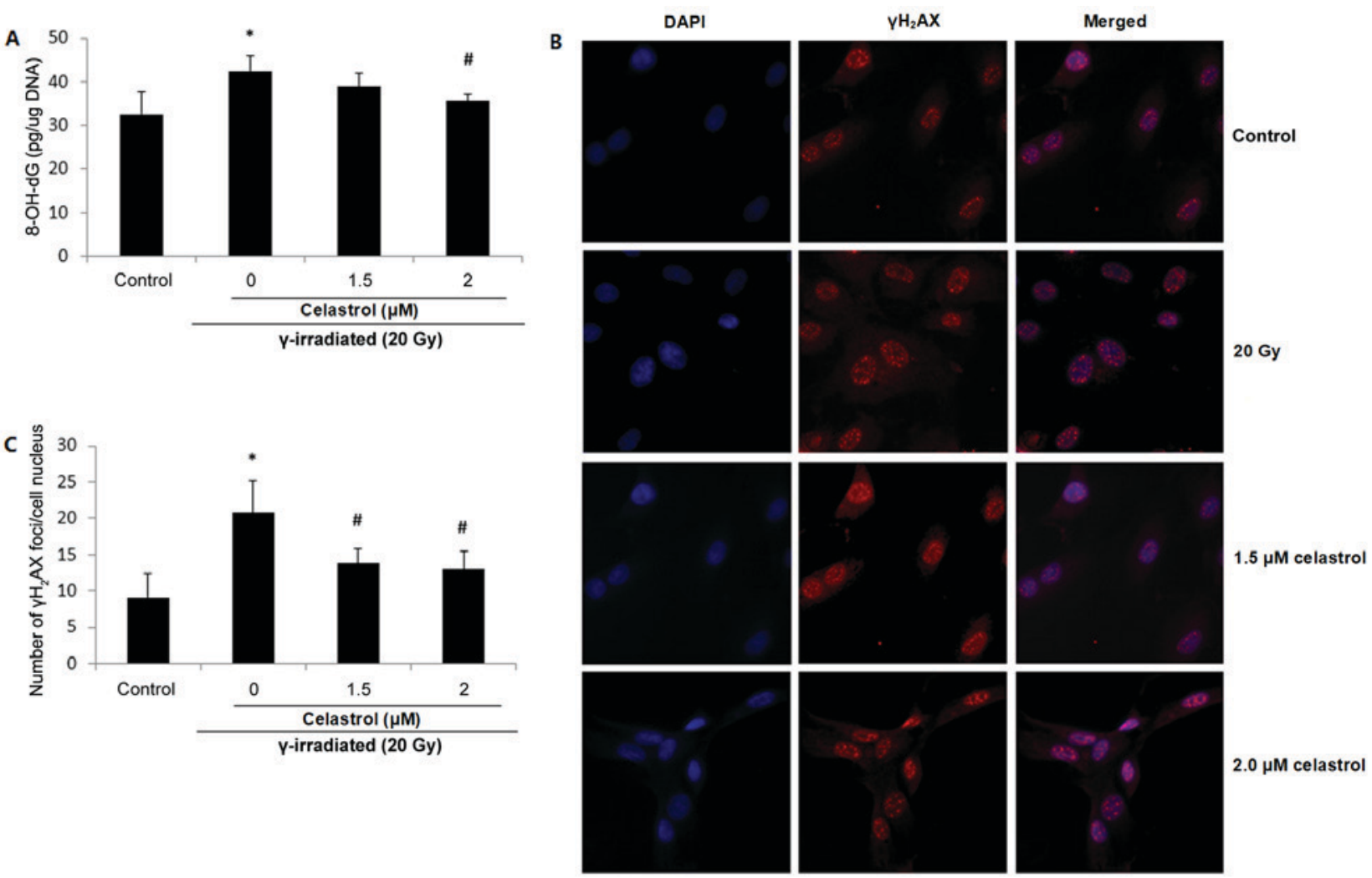

Figure 4. Effects of celastrol on DNA oxidative damage in HUVECs. Cells were exposed to 20-Gy $\gamma$ irradiation, followed by treatment with $1.5 \mu \mathrm{M}$ celastrol. HUVECs without celastrol treatment and $\gamma$ radiation served as the control. (A) Oxidative DNA damage was evaluated using an 8-OH-dG EIA kit. The absorbance at a wavelength of $420 \mathrm{~nm}$ was recorded and used to calculate the 8-OH-dG concentration based upon the standard curve. (B) Representative images of $\gamma \mathrm{H} 2 \mathrm{AX}$ fluorescence staining. DNA double-strand breaks were stained using anti- $\gamma \mathrm{H} 2 \mathrm{AX}$ antibody, and the nuclei were stained with DAPI. The images were examined under a fluorescence microscope (magnification, $\mathrm{x} 400$ ). (C) The number of $\gamma \mathrm{H} 2 \mathrm{AX}$ foci was counted and compared. All data are expressed as the mean \pm SEM. ANOVA was used to determine statistical significance between groups followed by Tukey's post-hoc test. ${ }^{*} \mathrm{P}<0.05$ vs. control; ${ }^{*} \mathrm{P}<0.05$ vs. $20 \mathrm{~Gy}$ without celastrol treatment group. HUVECs, human umbilical vein endothelial cells; 8-OH-dG, 8-hydroxy-2-deoxy Guanosine; ANOVA, one-way analysis of variance.

$\gamma$ irradiation induced oxidative damage in HUVECs, but also suggested that a suitable radioprotector should possess the ability to ameliorate oxidative stress, prevent peroxidation and restore the endogenous antioxidant system following radiation exposure.

Celastrol treatment was observed to exhibit protective effects in HUVECs in the present study by inhibiting ROS production, lipid peroxidation generation and oxidative DNA damage, indicative of the antioxidative stress properties of celastrol in radioprotection. Our results are in accordance with a report from Stankova et al (9), which demonstrated the antioxidative effects of celastrol in human peripheral blood mononuclear cells exposed to $\gamma$ radiation. The action of celastrol in attenuating oxidative stress has been investigated in cells and animal models of diabetes, colitis, atherosclerosis and hypertension (18,44-46). Furthermore, celastrol was demonstrated to be the most active compound among eight sesquiterpene esters in inhibiting $\mathrm{NF}-x \mathrm{~B}$ activation and NO production (47). The excessive production of NO in LPS-stimulated microglial cells was also inhibited by celastrol treatment (48). The inhibitory effect of celastrol on lipid peroxidation was demonstrated in rat mitochondria (49). The 

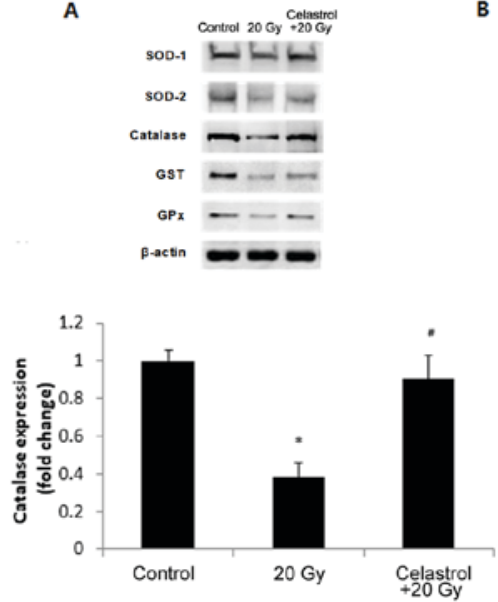
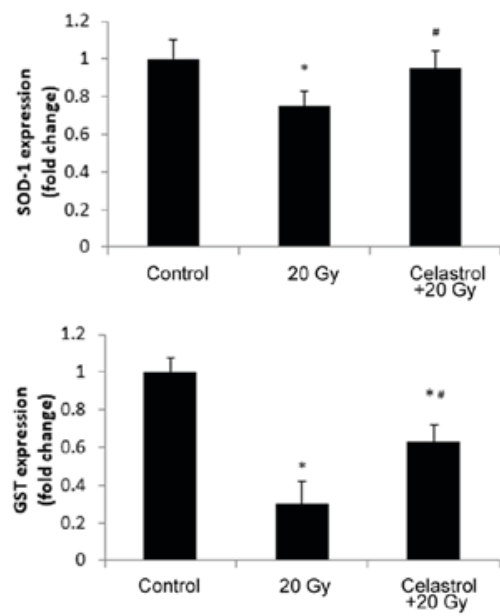
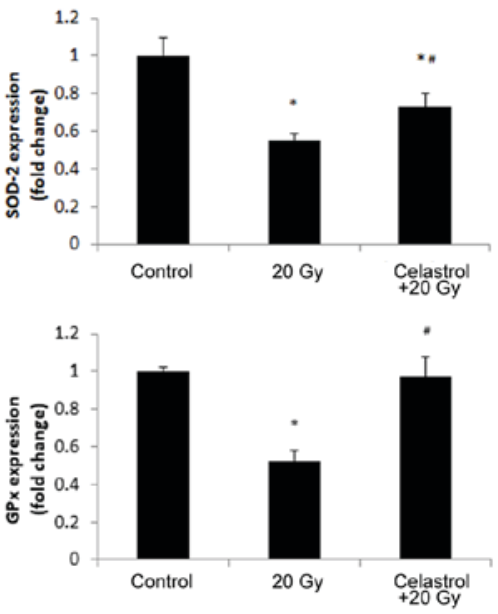

Figure 5. Effects of treatment with celastrol on the protein expression of antioxidant enzymes. Cells were exposed to 20-Gy $\gamma$ irradiation, followed by treatment with $1.5 \mu \mathrm{M}$ celastrol. HUVECs without celastrol treatment and $\gamma$ radiation served as the control. (A) Representative images of western blot analysis illustrating the intensities of SOD-1, SOD-2, catalase, GST, GPx and $\beta$-actin. (B) Densitometric analysis of band intensities of antioxidant enzymes. The image was captured and quantified using the Bio-Rad Gel Doc system. Values were normalized to the respective loading control ( $\beta$-actin). The results were calculated and expressed as a fold change relative to the control groups. Data are expressed as the mean \pm SEM. ANOVA was used to determine statistical significance between groups followed by Tukey's post-hoc test. " $\mathrm{P}<0.05$ vs. control; " $\mathrm{P}<0.05$ vs. 20 Gy without celastrol treatment group. HUVECs, human umbilical vein endothelial cells; SOD, superoxide dismutase; GPx, glutathione peroxidase; GST, glutathione S-transferase; ANOVA, one-way analysis of variance.

anti-peroxidative property of celastrol, together with its functions in the inhibition of ROS and NO production, supports our findings that celastrol suppressed $\gamma$ irradiation-induced oxidative stress in HUVECs.

ROS produced following $\gamma$ irradiation may act via the NADPH and xanthine oxidase pathways. NADPH oxidase is the major source of superoxide production. NADPH oxidase DUOX1 was reported to promote the long-term persistence of oxidative stress in a human thyroid cell line and primary thyrocytes after exposure to radiation (50). Xanthine oxidase is a type of enzyme that generates ROS and nitric oxide. It was demonstrated that exposure of rats to $\gamma$ radiation increased the levels of NO and xanthine oxidase activity, and decreased the GSH level, and SOD and CAT activity (51). Both pathways contribute to the generation of ROS following $\gamma$ radiation, which may negatively impact antioxidant defence mechanisms, reduce the intracellular concentrations of GSH and decrease the activities of antioxidant enzymes including SOD, catalase, GST and GPx. The imbalance in intracellular redox status will result in oxidative injury and cell death. In our study, 20-Gy $\gamma$ irradiation was demonstrated to significantly decrease the activities of antioxidant enzymes compared with the control. This decrease may be due to the elevated utilisation of the antioxidant system during the detoxification of $\gamma$ irradiation-induced free radicals. The treatment with celastrol was observed to reverse the decreased activities of antioxidant enzymes in HUVECs. The ability of celastrol to increased antioxidant capacities was also proven in pulmonary fibrosis and obesity $(52,53)$. Furthermore, HUVECs exhibited significantly decreased expression of SOD-1, SOD-2, catalase, GST, and GPx after 20-Gy $\gamma$ irradiation compared with the control. The treatment with celastrol completely restored the expression of SOD-1, catalase and GPX to the control levels, and partially recovered the protein levels of SOD-2 and GST. These protein expression results further support the antioxidative stress properties of celastrol in $\gamma$ irradiation-induced cell damage in HUVECs.
In summary, celastrol protects HUVECs against $\gamma$ radiation-induced cell death and migratory ability loss by decreasing ROS production and lipid peroxidation, mitigating oxidative DNA damage, and restoring the activity and protein expression of antioxidant enzymes. Given its antioxidative efficacy, pharmaceutical properties and low toxicity, celastrol may represent a potential novel agent in the protection against $\gamma$ irradiation-induced injury in endothelial cells.

\section{Acknowledgements}

Not applicable.

\section{Funding}

The present study was supported by Jilin Provincial Key Laboratory Program, the Research Foundation of Jilin Provincial Science and Technology Development (grant no. 20150622024JC).

\section{Availability of data and materials}

The datasets generated and/or analyzed during the current study are available from the corresponding author upon request.

\section{Authors' contributions}

FL and $\mathrm{XBH}$ conceived and designed the experiments, and performed the experiments. $\mathrm{XBH}, \mathrm{YQF}$ and $\mathrm{YT}$ analyzed the data. $\mathrm{XBH}$ and YQF contributed reagents/materials/analysis tools. XBH, YT, YQF and FL wrote the manuscript.

\section{Ethics approval and consent to participate}

Not applicable. 


\section{Patient consent for publication}

Not applicable.

\section{Competing interests}

The authors report no conflicts of interest. The authors alone are responsible for the content and writing of the paper.

\section{References}

1. Ungvari Z, Podlutsky A, Sosnowska D, Tucsek Z, Toth P, Deak F, Gautam T, Csiszar A and Sonntag WE: Ionizing radiation promotes the acquisition of a senescence-associated secretory phenotype and impairs angiogenic capacity in cerebromicrovascular endothelial cells: Role of increased DNA damage and decreased DNA repair capacity in microvascular radiosensitivity. J Gerontol A Biol Sci Med Sci 68: 1443-1457, 2013.

2. Yentrapalli R, Azimzadeh O, Barjaktarovic Z, Sarioglu H, Wojcik A, Harms-Ringdahl M, Atkinson MJ, Haghdoost S and Tapio S: Quantitative proteomic analysis reveals induction of premature senescence in human umbilical vein endothelial cells exposed to chronic low-dose rate gamma radiation. Proteomics 13: 1096-1107, 2013.

3. Young EF and Smilenov LB: Impedance-based surveillance of transient permeability changes in coronary endothelial monolayers after exposure to ionizing radiation. Radiat Res 176 415-424, 2011

4. Gabryś D, Greco O, Patel G, Prise KM, Tozer GM and Kanthou C: Radiation effects on the cytoskeleton of endothelial cells and endothelial monolayer permeability. Int J Radiat Oncol Biol Phys 69: 1553-1562, 2007

5. Park MT, Oh ET, Song MJ, Lee H and Park HJ: Radio-sensitivities and angiogenic signaling pathways of irradiated normal endothelial cells derived from diverse human organs. J Radiat Res 53 $570-580,2012$.

6. Thompson MA: Maintaining a proper perspective of risk associated with radiation exposure. J Nucl Med Technol 29: 137-142; quiz 148-150, 2001.

7. Doi H, Matsumoto S, Odawara S, Shikata T, Kitajima K, Tanooka M, Takada Y, Tsujimura T, Kamikonya N and Hirota S: Pravastatin reduces radiation-induced damage in normal tissues. Exp Ther Med 13: 1765-1772, 2017.

8. Kuchta K, Xiang Y, Huang S, Tang Y, Peng X, Wang X, Zhu Y, Li J, Xu J, Lin Z and Pan T: Celastrol, an active constituent of the TCM plant Tripterygium wilfordii Hook.f., inhibits prostate cancer bone metastasis. Prostate Cancer Prostatic Dis 20: 156-164, 2017.

9. Stankova K, Ivanova K, Nikolov V, Aneva N, Georgieva R and Boteva R: Proteasome inhibition protects human peripheral blood mononuclear cells from radiation-induced oxidative stress. Int J Radiat Biol 89: 493-500, 2013.

10. Jiang M, Zha Q, Zhang C, Lu C, Yan X, Zhu W, Liu W, Tu S, Hou L, Wang C, et al: Predicting and verifying outcome of Tripterygium wilfordii Hook F. based therapy in rheumatoid arthritis: From open to double-blinded randomized trial. Sci Rep 5: 9700, 2015.

11. Lv QW, Zhang W, Shi Q, Zheng WJ, Li X, Chen H, Wu QJ, Jiang WL, Li HB, Gong L, et al: Comparison of Tripterygium wilfordii Hook $\mathrm{F}$ with methotrexate in the treatment of active rheumatoid arthritis (TRIFRA): A randomised, controlled clinical trial. Ann Rheum Dis 74: 1078-1086, 2015.

12. Sun J, Shen X, Dong J, Wang H, Zuo L, Zhao J, Zhu W, Li Y, Gong J and Li J: Tripterygium wilfordii Hook F as maintenance treatment for Crohn's disease. Am J Med Sci 350: 345-351, 2015.

13. Zhu W, Li Y, Gong J, Zuo L, Zhang W, Cao L, Gu L, Guo Z, Li N and Li J: Tripterygium wilfordii Hook. f. versus azathioprine for prevention of postoperative recurrence in patients with Crohn's disease: A randomized clinical trial. Dig Liver Dis 47: 14-19, 2015.

14. Venkatesha SH and Moudgil KD: Celastrol and its role in controlling chronic diseases. Adv Exp Med Biol 928: 267-289, 2016.

15. Allison AC, Cacabelos R, Lombardi VR, Alvarez XA and Vigo C: Celastrol, a potent antioxidant and anti-inflammatory drug, as a possible treatment for Alzheimer's disease. Prog Neuropsychopharmacol Biol Psychiatry 25: 1341-1357, 2001.
16. Cleren C, Calingasan NY, Chen J and Beal MF: Celastrol protects against MPTP- and 3-nitropropionic acid-induced neurotoxicity. J Neurochem 94: 995-1004, 2005.

17. Venkatesha SH, Yu H, Rajaiah R, Tong L and Moudgil KD: Celastrus-derived celastrol suppresses autoimmune arthritis by modulating antigen-induced cellular and humoral effector responses. J Biol Chem 286: 15138-15146, 2011.

18. Yu X, Tao W, Jiang F, Li C, Lin J and Liu C: Celastrol attenuates hypertension-induced inflammation and oxidative stress in vascular smooth muscle cells via induction of heme oxygenase-1. Am J Hypertens 23: 895-903, 2010.

19. Abu Bakar MH, Sarmidi MR, Tan JS and Mohamad Rosdi MN: Celastrol attenuates mitochondrial dysfunction and inflammation in palmitate-mediated insulin resistance in C3A hepatocytes. Eur J Pharmacol 799: 73-83, 2017.

20. Liu J, Lee J, Salazar Hernandez MA, Mazitschek R and Ozcan U: Treatment of obesity with celastrol. Cell 161: 999-1011, 2015.

21. Avilla J, Teixidò A, Velázquez C, Alvarenga N, Ferro E and Canela R: Insecticidal activity of Maytenus species (Celastraceae) nortriterpene quinone methides against codling moth, Cydia pomonella (L.) (Lepidoptera: Tortricidae). J Agric Food Chem 48: 88-92, 2000.

22. Wang H, Sethi G, Loke WK and Sim MK: Des-aspartate-angiotensin i attenuates mortality of mice exposed to gamma radiation via a novel mechanism of action. PLoS One 10: e0138009, 2015.

23. Howard BJ, Fesenko S, Balonov M, Pröhl G and Nakayama S: A comparison of remediation after the Chernobyl and Fukushima Daiichi accidents. Radiat Prot Dosimetry 173: 170-176, 2017.

24. Ghosh SP, Kulkarni S, Perkins MW, Hieber K, Pessu RL, Gambles K, Maniar M, Kao TC, Seed TM and Kumar KS: Amelioration of radiation-induced hematopoietic and gastrointestinal damage by $\operatorname{Ex}-\mathrm{RAD}(\mathrm{R})$ in mice. J Radiat Res 53: 526-536, 2012.

25. Ran XZ, Ran X, Zong ZW, Liu DQ, Xiang GM, Su YP and Zheng HE: Protective effect of atorvastatin on radiation-induced vascular endothelial cell injury in vitro. J Radiat Res 51: 527-533, 2010.

26. El-Desouky W, Hanafi A and Abbas MM: Radioprotective effect of green tea and grape seed extracts mixture on gamma irradiation induced immune suppression in male albino rats. Int J Radiat Biol 93: 433-439, 2017.

27. Lata M, Prasad J, Singh S, Kumar R, Singh L, Chaudhary P, Arora R, Chawla R, Tyagi S, Soni NL, et al: Whole body protection against lethal ionizing radiation in mice by REC-2001: A semi-purified fraction of Podophyllum hexandrum. Phytomedicine 16: 47-55, 2009.

28. Sankhwar S, Gupta ML, Gupta V, Verma S, Suri KA, Devi M, Sharma P, Khan EA and Alam MS: Podophyllum hexandrum-mediated survival protection and restoration of other cellular injuries in lethally irradiated mice. Evid Based Complement Alternat Med 2011: 175140, 2011.

29. Dutta A, Verma S, Sankhwar S, Flora SJ and Gupta ML: Bioavailability, antioxidant and non toxic properties of a radioprotective formulation prepared from isolated compounds of Podophyllum hexandrum: A study in mouse model. Cell Mol Biol (Noisy-le-grand) 58 (Suppl): OL1646-OL1653, 2012.

30. Saini R, Verma S, Singh A and Lata Gupta M: Role of active principles of podophyllum hexandrum in amelioration of radiation mediated lung injuries by reactive oxygen/nitrogen species reduction. CellBio 2: pp105-116, 2013.

31. Sandeep D and Nair CK: Protection of DNA and membrane from $\gamma$-radiation induced damage by the extract of Acorus calamus Linn.: An in vitro study. Environ Toxicol Pharmacol 29: 302-307, 2010.

32. Sandeep D and Nair CK: Protection from lethal and sub-lethal whole body exposures of mice to $\gamma$-radiation by Acorus calamus L.: Studies on tissue antioxidant status and cellular DNA damage. Exp Toxicol Pathol 64: 57-64, 2012.

33. Tan H, Ashour A, Katakura Y and Shimizu K: A structure-activity relationship study on antiosteoclastogenesis effect of triterpenoids from the leaves of loquat (Eriobotrya japonica). Phytomedicine 22: 498-503, 2015.

34. Tang WJ, Wang J, Tong X, Shi JB, Liu XH and Li J: Design and synthesis of celastrol derivatives as anticancer agents. Eur J Med Chem 95: 166-173, 2015.

35. Wang Z, Zhai Z and Du X: Celastrol inhibits migration and invasion through blocking the NF- $\mathrm{B}$ pathway in ovarian cancer cells. Exp Ther Med 14: 819-824, 2017. 
36. Schultz-Hector S and Trott KR: Radiation-induced cardiovascular diseases: Is the epidemiologic evidence compatible with the radiobiologic data? Int J Radiat Oncol Biol Phys 67: 10-18, 2007.

37. Hwang S, Lee DH, Lee IK, Park YM and Jo I: Far-infrared radiation inhibits proliferation, migration, and angiogenesis of human umbilical vein endothelial cells by suppressing secretory clusterin levels. Cancer Lett 346: 74-83, 2014.

38. Sinha M, Das DK, Manna K, Datta S, Ray T, Sil AK and Dey S: Epicatechin ameliorates ionising radiation-induced oxidative stress in mouse liver. Free Radic Res 46: 842-849, 2012.

39. Yu J, Zhu X, Qi X, Che J and Cao B: Paeoniflorin protects human EA.hy926 endothelial cells against gamma-radiation induced oxidative injury by activating the NF-E2-related factor 2/heme oxygenase-1 pathway. Toxicol Lett 218: 224-234, 2013.

40. Yu J, Piao BK, Pei YX, Qi X and Hua BJ: Protective effects of tetrahydropalmatine against gamma-radiation induced damage to human endothelial cells. Life Sci 87: 55-63, 2010.

41. Hu S, Gao Y, Zhou H, Kong F, Xiao F, Zhou P and Chen Y: New insight into mitochondrial changes in vascular endothelial cells irradiated by gamma ray. Int J Radiat Biol 93: 470-476, 2017.

42. Cervelli T, Panetta D, Navarra T, Andreassi MG, Basta G, Galli A Salvadori PA, Picano E and Del Turco S: Effects of single and fractionated low-dose irradiation on vascular endothelial cells. Atherosclerosis 235: 510-518, 2014.

43. Olteanu D, Baldea I, Clichici S, Bolfa P, Cenariu M, Schrepler-Perde M, Alupei M, Muresan A and Filip A: In vitro studies on the mechanisms involved in chemoprevention using Calluna vulgaris on vascular endothelial cells exposed to UVB. J Photochem Photobiol B 136: 54-61, 2014.

44. Gu L, Bai W, Li S, Zhang Y, Han Y, Gu Y, Meng G, Xie L, Wang J, Xiao Y, et al: Celastrol prevents atherosclerosis via inhibiting LOX-1 and oxidative stress. PLoS One 8: e65477, 2013.

45. Guan Y, Cui ZJ, Sun B, Han LP, Li CJ and Chen LM: Celastrol attenuates oxidative stress in the skeletal muscle of diabetic rats by regulating the AMPK-PGC1 $\alpha$-SIRT3 signaling pathway. Int J Mol Med 37: 1229-1238, 2016.

46. Shaker ME, Ashamallah SA and Houssen ME: Celastrol ameliorates murine colitis via modulating oxidative stress, inflammatory cytokines and intestinal homeostasis. Chem Biol Interact 210: 26-33, 2014
47. Jin HZ, Hwang BY, Kim HS, Lee JH, Kim YH and Lee JJ: Antiinflammatory constituents of Celastrus orbiculatus inhibit the NF-kappaB activation and NO production. J Nat Prod 65: 89-91, 2002.

48. Jung HW, Chung YS, Kim YS and Park YK: Celastrol inhibits production of nitric oxide and proinflammatory cytokines through MAPK signal transduction and NF-kappaB in LPS-stimulated BV-2 microglial cells. Exp Mol Med 39: 715-721, 2007.

49. Sassa H, Takaishi Y and Terada H: The triterpene celastrol as a very potent inhibitor of lipid peroxidation in mitochondria. Biochem Biophys Res Commun 172: 890-897, 1990.

50. Ameziane-El-Hassani R, Talbot M, de Souza Dos Santos MC, Al Ghuzlan A, Hartl D, Bidart JM, De Deken X, Miot F, Diallo I, de Vathaire F, et al: NADPH oxidase DUOX1 promotes long-term persistence of oxidative stress after an exposure to irradiation. Proc Natl Acad Sci USA 112: 5051-5056, 2015.

51. Shaban NZ, Ahmed Zahran AM, El-Rashidy FH and Abdo Kodous AS: Protective role of hesperidin against $\gamma$-radiation-induced oxidative stress and apoptosis in rat testis. J Biol Res (Thessalon) 24: 5, 2017.

52. Divya T, Dineshbabu V, Soumyakrishnan S, Sureshkumar A and Sudhandiran G: Celastrol enhances Nrf2 mediated antioxidant enzymes and exhibits anti-fibrotic effect through regulation of collagen production against bleomycin-induced pulmonary fibrosis. Chem Biol Interact 246: 52-62, 2016.

53. Wang C, Shi C, Yang X, Yang M, Sun H and Wang C: Celastrol suppresses obesity process via increasing antioxidant capacity and improving lipid metabolism. Eur J Pharmacol 744: 52-58, 2014.

This work is licensed under a Creative Commons Attribution-NonCommercial-NoDerivatives 4.0 International (CC BY-NC-ND 4.0) License. 